\title{
A HIGH-RESOLUTION POLARIZATION SURVEY OF THE NORTH POLAR SPUR
}

\author{
G. WESTERHOUT and D. BECHIS \\ University of Maryland, College Park, Md., U.S.A.
}

\begin{abstract}
Observations have been made at $21 \mathrm{~cm}$ with a resolution of $11^{\prime}$ to look for fine structure in the polarization distribution. In the North Polar Spur, the angular scale of the polarization parameters varies with latitude. This is attributed to an increase in the irregularity of the magnetic field in the Spur with latitude.
\end{abstract}

\section{Introduction}

Following the extensive polarization observations by the Dutch group, notably Brouw and Spoelstra (1974) and Spoelstra $(1971,1972)$, it seemed appropriate to try and find out whether a resolution of $10^{\prime}$ would reveal smaller structures in certain regions where the polarization distribution is still rather complex, even at $21 \mathrm{~cm}$ with a $36^{\prime}$ beam. Setting a limit to the size of the irregularities in the magnetic field in a region such as the North Polar Spur might help in deciding what mechanism is causing depolarization of the radio radiation in this direction and what mechanism is generating the intense synchrotron emission observed in this presumably old supernova remnant. It might also provide a measure of the 'clumpiness' of the medium.

Observations were made over a total of about 75 days spread over several periods between June 1971 and March 1972, with the 300-ft transit telescope of the National Radio Astronomy Observatory* in Green Bank, West Virginia, at a wavelength of $21 \mathrm{~cm}$ where the beamwidth is approximately $11^{\prime}$. At this time, we have only reduced the region of the North-Polar Spur, i.e. between Dec. $6^{\circ}-16^{\circ}$, R.A. 15:45-19:00 $\left(b=5^{\circ}-55^{\circ}, l=45^{\circ}-15^{\circ}\right)$. Points were observed every $10^{\prime}$ in declination and every $6^{\prime}$ in right ascension.

\section{Observational Results}

The following conclusions may be drawn from the observations:

(1) The scale over which the intensity of the polarized radiation and its position angle remain uniform varies with latitude:

$b$

$$
\begin{array}{ll}
55^{\circ} & \sim 3^{\circ} \text { or more } \\
35^{\circ} & \sim 1^{\circ} \text { to } 2^{\circ} \\
15^{\circ} & \sim 0.5^{\circ} \text { to } 1^{\circ}
\end{array}
$$

If there are any polarization features smaller than the $11^{\prime}$ beamwidth, they exist mostly

* The National Radio Astronomy Observatory is operated by Associated Universities Inc. under contract with the National Science Foundation. 
at lower latitudes in the Spur. However, over the most intense regions of the Spur there do not seem to be any features with angular size less than 0:5. (2) Measurements over the brightest points of the Spur show that the degree of polarization (calculated by extrapolating Berkhuijsen's (1971) $820-\mathrm{MHz}$ map to $1400 \mathrm{MHz}$ using a spectral index of -2.7 ) decreases with latitude from about $70 \%$ for $b>40^{\circ}$ to about $35 \%$ around $b=20^{\circ}$. (3) The low polarization intensities measured in the Spur for $b<+50^{\circ}$ by the Dutch surveys between 408 and $820 \mathrm{MHz}$ may well be due partly to averaging smallscale polarization features over their $2^{\circ}$ to $1^{\circ}$ telescope beam. But this cannot explain the 'depolarization' of the $1400-\mathrm{MHz}$ radiation at low latitudes as polarization structure much smaller than 0.5 does not seem to be present over the most intense regions of the Spur.

\section{Discussion}

Three possible explanations exist. The depolarization could be due to differential Faraday rotation caused by random fluctuations along the line of sight in the magnetic field and/or the electron density either (1) in the intervening medium, or (2) in the Spur itself. On the other hand, (3) random fluctuations in the Spur's magnetic field alone will tend to lower the intrinsic degree of polarization of the radiation emitted from the Spur. Berkhuijsen (1971) has considered these explanations in some detail. The present survey, because of its high angular resolution, adds one important piece of information: the size of the irregularities. We take the angular size of uniformity to be about $1^{\circ}$ for $b<50^{\circ}$, corresponding to a linear size of a 'cell' of uniform magnetic field of about $1.6 \mathrm{pc}$, since the Spur's emission regions are at a distance of at most 100 pc. Using this figure we shall now discuss the three possible explanations; our conclusions are similar to those of Berkhuijsen.

Burn (1966) has shown that in the case that the depolarization occurs in the intervening medium, the degree of polarization we observe varies as

$$
P\left(\lambda^{2}\right)=p_{\mathrm{i}} \exp \left\{-2 K^{2}\left(n_{\mathrm{e}} H_{\mu}\right)_{\mathrm{f}}^{2} \lambda^{4} R d\right\}
$$

Here, $p_{\mathrm{i}}$ is the initial degree of polarization, $d$ is the size of a cell in which the fluctuation occurs, $\left(n_{\mathrm{e}} H_{\|}\right)_{\mathrm{f}}^{2}$ is the variance of the product of the electron density and the line-ofsight magnetic field of a cell, $R$ is the path length through the intervening medium, and $K=2.62 \times 10^{-17}$, with all quantities in cgs units. For significant depolarization to occur, the quantity in brackets must be at least unity, which means that with $R=100 \mathrm{pc}$ and $d=1.6$ pc we have $\left(n_{\mathrm{e}} H_{\|}\right)_{\mathrm{f}} \gtrsim 2 \times 10^{-6} \mathrm{G} \mathrm{cm}^{-3}$. Assuming a random component of $H_{\|}$as high as the mean value observed in the interstellar medium, $\sim 3 \times 10^{-6} \mathrm{G}$, we find that a mean value of the electron density $n_{\mathrm{e}} \gtrsim 1 \mathrm{~cm}^{-3}$ is needed to explain the depolarization. Since this is far in excess of the average electron density in the interstellar medium, which is generally assumed to be $<0.1 \mathrm{~cm}^{-3}$, we can rule out depolarization in the intervening medium.

Let us now see whether differential Faraday rotation within the Spur's emission regions can account for the depolarization. Since Burn's calculations are more complex in this case, we will deal with them in a more simplified way. Because the 
pathlength $L$ through the Spur's emission regions is of the same order as their distance, $100 \mathrm{pc}$, each line of sight passes through at most $N \sim 60$ cells, each of length $1.6 \mathrm{pc}$. For the case where $N \gg\left(H_{\mathrm{r}} / 2 H_{0}\right)^{2}$, with $H_{\mathrm{r}}$ and $H_{0}$ equal to the magnitudes of the random and the uniform components of the magnetic field, respectively, Burn calculates

$$
P\left(\lambda^{2}\right)=p_{\mathrm{i}} \frac{\sin \delta}{\delta}, \quad \text { where } \quad \delta=K n_{\mathrm{e}} H_{0} L \lambda^{2}
$$

Significant depolarization will occur only when $\delta>1$, corresponding to rotation measures in excess of 24 radians $\mathrm{m}^{-2}$ at $21 \mathrm{~cm}$. Since the rotation measures calculated by Spoelstra (1971) are no greater than 15 radians $\mathrm{m}^{-2}$ for latitudes between $b=+25^{\circ}$ and $b=+49^{\circ}$ in the Spur, it is hard to explain the high degrees of polarization (around $40 \%$ ) observed here. It is possible that if rotation measures could have been calculated on a closer angular spacing in this part of the sky, rotation measures in excess of 24 radians $\mathrm{m}^{-2}$ might have been found. However, Berkhuijsen (1971) finds in the Spur that the degree of polarization at $820 \mathrm{MHz}$ is always about 0.4 to 0.6 times that at $1411 \mathrm{MHz}$ over a wide range in degree of polarization. Such behavior would not be anticipated if differential Faraday rotation were an important factor in causing depolarization. Therefore, this model also fails to account for the latitude-dependent depolarization seen in the Spur.

If, however, the random fluctuations around the general direction of the magnetic field inside the Spur's emission regions increase toward lower latitudes, all observations are satisfied simply. Moreover, we can obtain an estimate of the strength of the random component of the magnetic field. For the case in which a random field $H_{\mathrm{r}}$ is superimposed on a uniform field $H_{0}$ and $H_{0} \gg H_{\mathrm{r}}$, Ginzburg and Syrovatskii (1965) give

$$
\Pi=\left[1-\frac{2}{3}\left(\frac{H_{\mathrm{r}}}{H_{0}}\right)^{2}\right] \frac{\gamma+1}{\gamma+7 / 3}
$$

for the degree of polarization of radiation of spectral slope $\gamma$ passing out of the source. Since the Spur has $\gamma \approx-2.7$ (Berkhuijsen, 1971), the gas giving rise to radiation with a a polarization of $35 \%$ has $H_{\mathrm{r}} \sim 0.8 H_{0}$. Berkhuijsen (1971) found $H_{\mathrm{r}} \approx 1.2 H_{0}$ from data at $829 \mathrm{MHz}$ with a beamwidth of $1^{\circ}$ at $b=+40^{\circ}$ in the Spur. Of course for $H_{0} \approx H_{\mathrm{r}}$ this expression no longer holds, but the simple calculation gives at least a rough estimate. It is interesting that Jokipii and Lerche (1969), using Faraday rotations of extragalactic sources and dispersion measures of pulsar signals, found a fluctuating magnetic field of $1.4 \times 10^{-6} \mathrm{G}$, about half the magnitude of the regular field, $\approx 3 \times 10^{-6} \mathrm{G}$.

We conclude then that Faraday rotation is unimportant in causing the decrease of the degree of polarization with galactic latitude in the Spur. It appears that instead the irregularity of the magnetic field in the Spur increases with decreasing latitude and that cells of constant magnetic field have a size of the order of $1.6 \mathrm{pc}$ at intermediate latitudes. It seems somewhat unsatisfactory that in such a local phenomenon as the Spur there would be a latitude dependence. However, it should be noted that the 
intensity of the total radiation from the Spur also increases with decreasing latitude, at least down to $b=+15^{\circ}$. Therefore, it might well be that the field irregularities are somehow related to the intensity of the radiation (or vice versa).

\section{Acknowledgement}

Part of this work was supported by the National Science Foundation.

\section{References}

Berkhuijsen, E. M.: 1971, Astron. Astrophys. 14, 359.

Brouw, W. N. and Spoelstra, T. A. Th.: 1974, Astron. Astrophys., submitted.

Burn, B. J.: 1966, Monthly Notices Roy. Astron. Soc. 133, 67.

Ginzburg, V. L. and Syrovatskii, S. I.: 1965, Ann Rev. Astron. Astrophys. 3, 297.

Jokipii, J. R. and Lerche, I.: 1969, Astrophys. J. 157, 1137.

Spoelstra, T. A. Th.: 1971, Astron. Astrophys. 13, 237.

Spoelstra, T. A. Th.: 1972, Astron. Astrophys. Suppl. 7, 169.

\section{G. Westerhout \\ D. Bechis}

Astronomy Program, University of Maryland,

College Park, Md. 20742, U.S.A.

\section{DISCUSSION}

Baldwin: Are the percentage polarizations you quote relative to the total continuum emission or to a notional spur contribution obtained by some subtraction procedure? And do you feel secure about the variation with latitude?

Westerhout: The percentage polarization is derived by extrapolating Berkhuijsen's $820 \mathrm{MHz}$ total intensity map using a spectral index of -2.7 . Therefore, they are very uncertain (I estimate $\pm 30 \%$ ) and we did not subtract a background. However, I feel quite sure about the decrease in degree of polarization with decreasing latitude (or, alternatively, with increasing intensity in the Spur). This has of course already been noted and discussed extensively by other authors, notably Berkhuijsen and Spoelstra.

Wielibinski: In the narrow neck of the Spur there is certainly structure on a smaller scale than described in this paper. On a continuum map made at $11 \mathrm{~cm}$ by Baker, Berkhuijsen and Haslam there is structure on the scale of some $5^{\prime}$.

Westerhout: Unfortunately, we only have limited coverage of the narrow neck; these data have not yet been reduced, and it will be very interesting indeed to see whether the polarization structure in the narrow neck will be smaller than the 0.5 at higher latitudes. 